\title{
DNA oxidative damage of neoplastic rat liver lesions
}

\author{
BARBARA MARENGO ${ }^{*}$, CHIARA DE CIUCIS ${ }^{2 *}$, ROBERTA RICCIARELLI $^{2}$, PAOLA ROMANO $^{2}$, \\ MARIO PASSALACQUA ${ }^{3}$, UMBERTO M. MARINARI ${ }^{2}$, MARIA A. PRONZATO $^{2}$ and CINZIA DOMENICOTTI ${ }^{2}$ \\ ${ }^{1}$ Giannina Gaslini Institute, Genoa; ${ }^{2}$ Department of Experimental Medicine, Section of General Pathology, ${ }^{3}$ Department of \\ Experimental Medicine, Section of Biochemistry, Centre of Excellence in Biomedical Research, University of Genoa, Italy
}

Received November 13, 2009; Accepted December 30, 2009

DOI: 10.3892/or_00000756

\begin{abstract}
Hepatic lesions, experimentally-induced in Fisher 344 (F344) and Brown Norway (BN) rats, respectively, susceptible and resistant to liver carcinogenesis, progress differently to hepatocellular carcinoma (HCC). The mechanisms responsible for the acquisition of the resistant phenotype are not completely clear. Herein, we show that in F344 rats subjected to carcinogenic treatment, angiogenesis and DNA oxidation markers increase in preneoplastic and neoplastic liver lesions. On the contrary, in the HCCs of treated $\mathrm{BN}$ rats, angiogenesis and a minor DNA oxidation are accompanied by an attempt of tissue remodelling. This study suggests that DNA oxidation might be an important factor in the initiation and promotion of the events of hepatocarcinogenesis. On the other hand, the enhancement of GSH levels and the down-regulation of superoxide dismutase (SOD) expression in both rat strains suggest that antioxidant response is not involved in the acquisition of resistant phenotype.
\end{abstract}

\section{Introduction}

Liver carcinogenesis is a multi-stage process (1) and several experimental models have been used to study the different steps of hepatocellular carcinoma (HCC) progression. Recently, it has been demonstrated that Fisher 344 (F344) and Brown Norway (BN) rats progress differently to HCC (2). In F344 animals, neoplastic lesions are characterised by rapid growth and progression to more malignant stages whereas the majority of lesions in BN rats grow very slowly and do not progress to full malignancy. Thus, inter-strain comparison of the different lesions could give insight into the relationships between molecular features and tumour progression rate.

Correspondence to: Dr Cinzia Domenicotti, Department of Experimental Medicine, Section of General Pathology, University of Genoa, Via L.B. Alberti 2, 16132 Genoa, Italy

E-mail: cdomeni@medicina.unige.it

${ }^{*}$ Contributed equally

Key words: hepatocellular carcinoma, DNA oxidative damage, glutathione
Important studies have shown an association between hepatocarcinogenesis and oxidative DNA damage by demonstrating the suppression of carcinogenesis upon the administration of antioxidant therapies $(3,4)$. 8-Hydroxy-2'-deoxyguanosine (8-OHdG) is a good marker of DNA oxidation and has been demonstrated to be implicated in hepatocarcinogenesis (5).

Cellular glutathione (GSH) and related enzymes work as the main antioxidant protective mechanisms against reactive oxygen species (ROS) and free radicals (6) and changes in GSH metabolism have been implicated in tumour progression (7). Copper-zinc superoxide dismutase $(\mathrm{Cu}, \mathrm{Zn}-\mathrm{SOD}$; SOD1) and manganese containing SOD (Mn-SOD; SOD2) synergize with the GSH redox system in order to provide cellular defence (8).

The aim of the present study was to monitor the presence of $8-\mathrm{OHdG}$ in the foci of altered hepatocytes, preneoplastic and neoplastic nodules in F344 and BN rat strains. Furthermore, following the steps of experimentallyinduced HCC, the levels of GSH and SOD as parameters of the antioxidant status, are to be determined.

\section{Materials and methods}

Liver samples. Frozen-stored liver samples were kindly provided by Professor F. Feo (University of Sassari, Italy). In the laboratory of Professor Feo, male Wistar F344 and BN rats were treated with DENA/AAF/partial hepatectomy (9), according to the 'resistant hepatocyte' $(\mathrm{RH})$ protocol (10).

Histology. Frozen livers of F344 and BN rats were sectioned $(5 \mu \mathrm{m})$ on a cryostat (Leica Microsystems AG, Wetzlar, Germany), placed on microscope polylysine coated slides, fixed in $10 \%$ formalin and stained with hematoxylin and eosin.

Analyses of apoptotic nuclei in liver cryosections were performed by TdT-FragEL ${ }^{\mathrm{TM}}$ DNA fragmentation detection kit (Calbiochem, La Jolla, CA). Briefly, frozen liver sections were fixed in $4 \%$ paraformaldehyde and permeabilised with $2 \mathrm{mg} / \mathrm{ml}$ Proteinase $\mathrm{K}$ in $10 \mathrm{mM}$ Tris $\mathrm{pH}$ 8.0. The endogenous peroxidases were inactivated by a solution of $3 \%$ $\mathrm{H}_{2} \mathrm{O}_{2}$ in methanol. Then, slides were incubated with a $5 \mathrm{X}$ Equilibration buffer and subsequently labelled with TdT Labelling Reaction Mixture. After reaction with Blocking buffer, slides were stained with a solution of 3,3' diaminobenzidine $(0.7 \mathrm{mg} /$ tablet $)$ and $\mathrm{H}_{2} \mathrm{O}_{2} /$ urea and counterstained 
A)

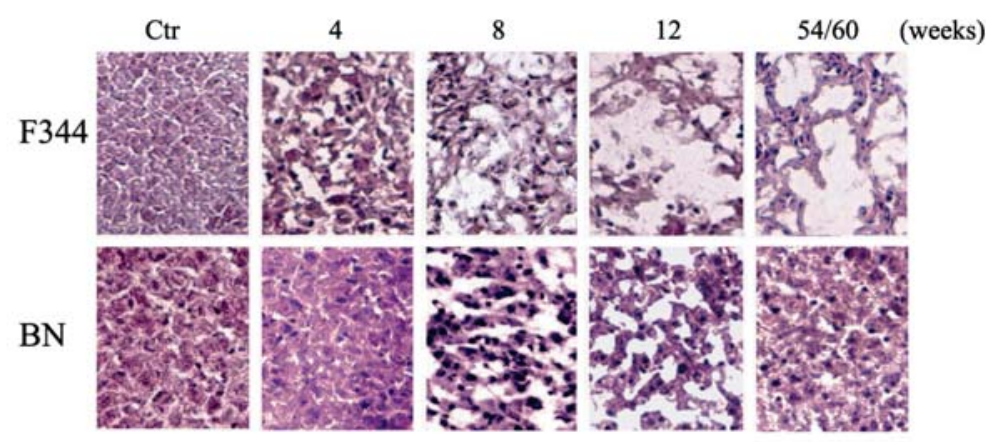

B)

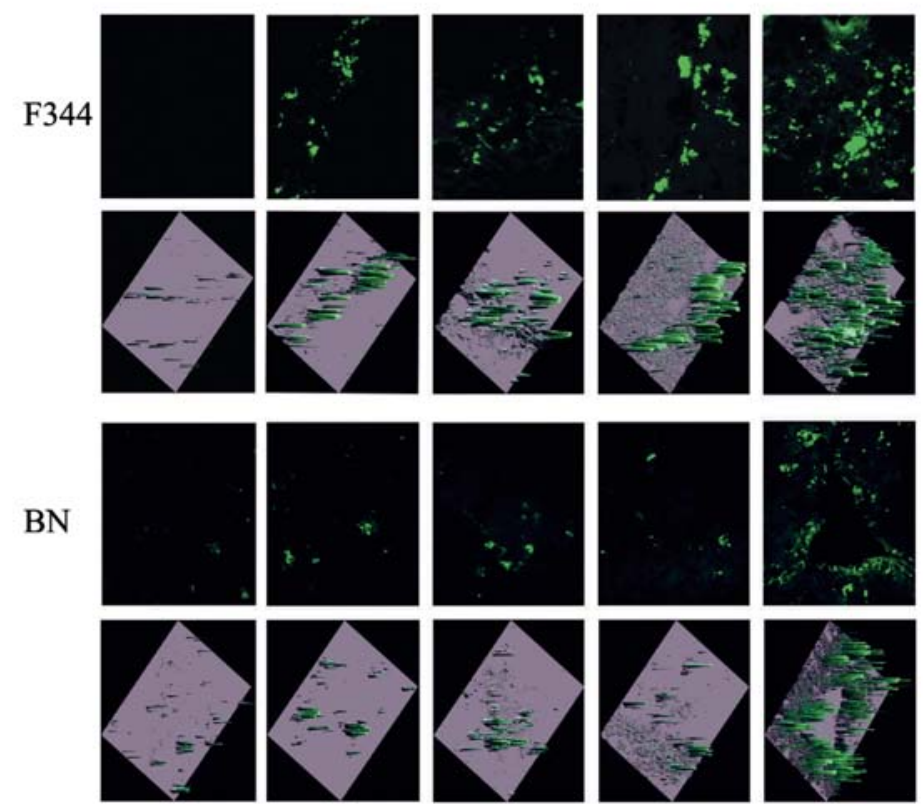

Figure 1. (A) Hematoxylin-eosin staining of liver sections from F344 and BN rats. For both strains, representative microphotographs of hepatic tissues from untreated (Ctr) rats and from animals after carcinogenic treatment $(4,8,12,54 / 60$ weeks) are shown. Magnification, x10. (B) Immunofluorescence analysis of vascular endothelial growth factor (VEGF). For both strains of rats (F344 and BN), representative microphotographs of fluorescently-labelled VEGF in liver sections from untreated (Ctr) and treated $(4,8,12$, and 54/60 weeks) rats are shown (upper panels). Magnification, x40. The lower panels show surface plots for the fluorescent images reported in the upper panels. Intensity spikes from each individual pixel can be seen.

with methyl green. After rinsing in ethanol and xylene, the slides were mounted with Cristal mount (Bio-Optica, Milan, Italy) and observed by Leica DMIRB microscope (Leica), using a standard set of filters and a $10 \mathrm{X}$ objective.

Immunofluorescence analysis. Frozen livers of F344 and BN rats, sectioned $(5 \mu \mathrm{m})$ on a cryostat (Leica), were placed on a microscope polylysine coated slide, fixed in $4 \%$ paraformaldehyde in PBS (137 mM NaCl, $2.6 \mathrm{mM} \mathrm{KCl}, 10 \mathrm{mM}$ $\mathrm{Na}_{2} \mathrm{HPO}_{4}, 1.8 \mathrm{mM} \mathrm{KH} \mathrm{PO}_{4} \mathrm{pH}$ 7.4) and permeabilised with $0.1 \%$ Triton $\mathrm{X}-100$. Mouse monoclonal antibody anti-8OHdG (1:500; Trevigen, Gaithersburg, MD) and antivascular endothelial growth factor (VEGF, 1: 240; Abcam Cambridgeshire, UK) were used as primary antibodies. FITC-conjugated secondary antibody (Upstate, DBA, Milan, Italy) was added at the same concentration of the primary antibodies. Slides were mounted with Gel Mount (BioOptica, Milan, Italy) and analysed by Leica DMIRB microscope using a standard set of filters for fluorescein and a 40X oil-immersion objective. After acquisition, the fluorescence intensity was analysed by Laser Pix Software (BioRad, Milan, Italy).
Reduced and oxidized glutathione levels. Liver homogenate was prepared in ice-cold lysis buffer containing $154 \mathrm{mM}$ $\mathrm{KCl}, 5 \mathrm{mM}$ diethylenetriaminepentaacetic acid (DTPA) and $0.1 \mathrm{M}$ potassium phosphate buffer, $\mathrm{pH}$ 6.8. An aliquot was removed for the determination of protein concentration (Lowry method). Subsequently, one volume of cold acid buffer containing $40 \mathrm{mM} \mathrm{HCl}, 10 \mathrm{mM}$ DTPA, $20 \mathrm{mM}$ ascorbic acid, and 5\% trichloracetic acid (TCA), was added to one volume of homogenate. The suspension was centrifuged at $14000 \mathrm{x} \mathrm{g}$ and the resulting supernatant was centrifuged through a $0.45 \mu \mathrm{m}$ microcentrifuge filter, yielding a $5 \%$ deproteinised homogenate, in which GSH and GSSG contents were determined by reaction with o-phthalaldehyde (OPA) (11). Standard solutions were prepared by dissolving GSH or GSSG in Redox Quenching Buffer (RQB) containing $20 \mathrm{mM} \mathrm{HCl}, 5 \mathrm{mM}$ DTPA and $10 \mathrm{mM}$ ascorbic acid. Deproteinised homogenate, or standards (GSH or GSSG), were treated with 5\% TCA-RQB solution. To evaluate GSSG levels, N-ethylmaleimide (7.5 mM in RQB) was added. Subsequently, $1 \mathrm{M}$ potassium phosphate $\left(\mathrm{KP}_{\mathrm{i}}\right)$ buffer $(\mathrm{pH}$ 7.0) was added to all tubes and the samples were incubated for $5 \mathrm{~min}$ at room temperature. For GSSG assay $100 \mathrm{mM}$ 
A)
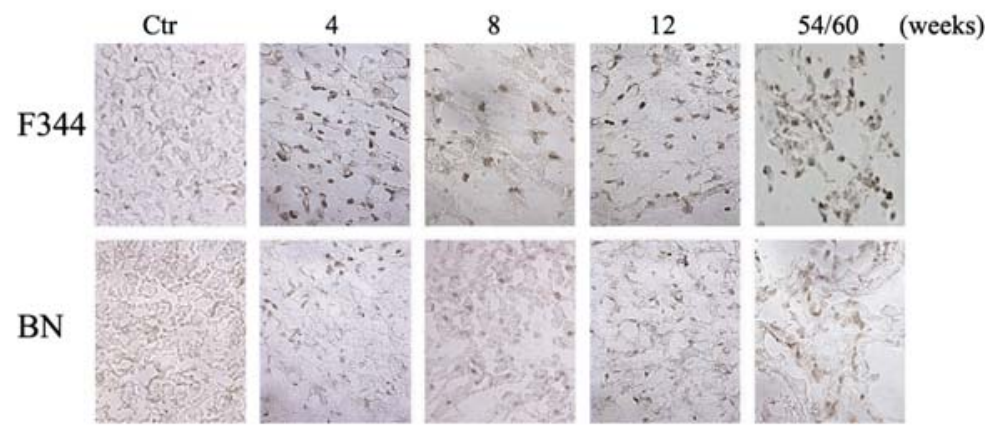

B)

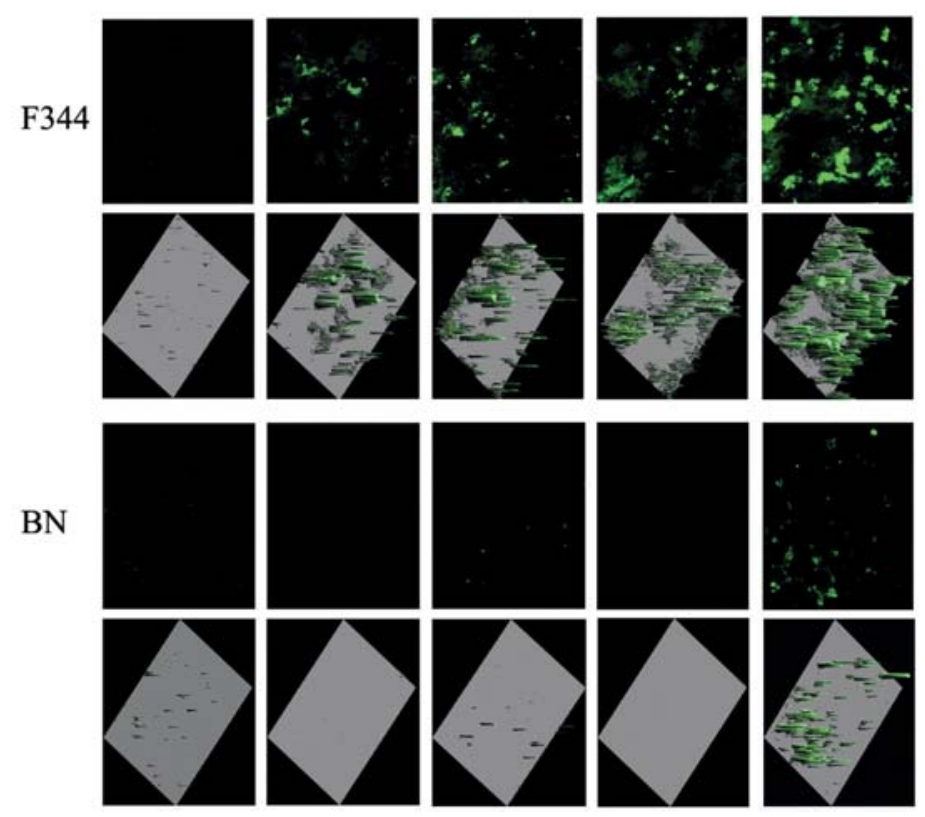

Figure 2. (A) Histological determination of apoptosis by Tdt-mediated UTP nick-end labeling (TUNEL) staining. Representative images of TUNEL staining in normal liver (Ctr) and in livers of rats treated (4, 8, 12 and 54/60 weeks). Cells, positive for TUNEL labelling, show brown nuclei which reflect DNA fragmentation characteristic of apoptosis. Magnification, x10. (B) Immunofluorescence analysis of 8-Hydroxy-2'-deoxy-guanosine (8-OHdG). For both strains, (F344 and BN) representative images of fluorescent 8-OHdG were taken at the indicated time of tumorigenic treatment (upper panels). Magnification, $\mathrm{x} 40$. The lower panels show surface plots for the fluorescent images reported in the upper panels. Intensity spikes from every individual pixel can be seen.

dithionite-RQB was used and the homogenates were incubated for $60 \mathrm{~min}$ at room temperature. Then, $0.1 \mathrm{M} \mathrm{KP_{i }}$ buffer ( $\mathrm{pH} 6.9)$ and OPA $(5 \mathrm{mg} / \mathrm{ml}$ in methanol) were added and, after a 30-min incubation at room temperature, sample fluorescence was monitored with a Perkin Elmer fluorimeter (Perkin Elmer Life and Analytical Sciences, Shelton, USA) at $365 / 430 \mathrm{~nm}$.

Immunoblot analysis. Liver samples from F344 and BN rats were homogenised in lysis buffer containing $10 \mathrm{mM}$ Hepes, $0.25 \mathrm{M}$ sucrose, $5 \mathrm{M}$ EDTA, $10 \mathrm{mM}$ ß mercaptoethanol, $0.3 \%$ Triton $\mathrm{X}-100,0.3 \%$ protease inhibitors

Immunoblots were carried out according to standard methods, using polyclonal rabbit anti-SOD1 and monoclonal mouse anti-SOD2 (AbCam, Cambridgeshire, UK); monoclonal mouse anti- $\beta$-actin (Sigma); anti-mouse and antirabbit secondary antibodies coupled to horseradishperoxidase (Amersham International, Buckinghamshire, UK). Proteins were visualised with an enzyme-linked chemiluminescence detection kit according to the manufacturer's instructions (Amersham). Chemiluminescence was monitored by exposure to film and the signals were analysed, under non-saturating conditions, with an image densitometer connected to Quantity One software (BioRad laboratories, Hercules, CA, USA).

Data analysis. Results were expressed as mean \pm SD from at least three independent experiments. The statistical significance of parametric differences among sets of experimental data was evaluated by one-way ANOVA and Bonferroni's test for multiple comparisons.

\section{Results}

Microscopy analysis of hematoxylin-eosin stained liver sections of rats showed a progressive reduction of liver parenchyma which, in F344 rats, was already appreciated at 4 weeks from the tumorigenic treatment. Impressively, after 54 weeks, the characteristic liver parenchyma structure was totally destroyed. In $\mathrm{BN}$ rats, atrophy of the hepatic tissue started at 8 weeks whereas a partial attempt of remodelling was evident at 60 weeks (Fig. 1A). 
A)

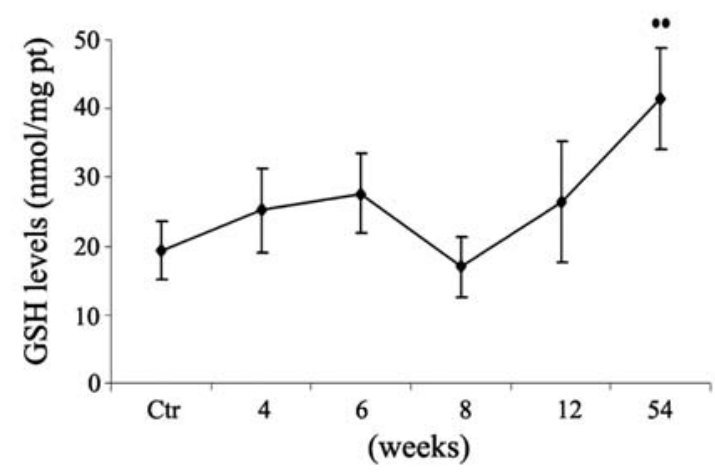

B)

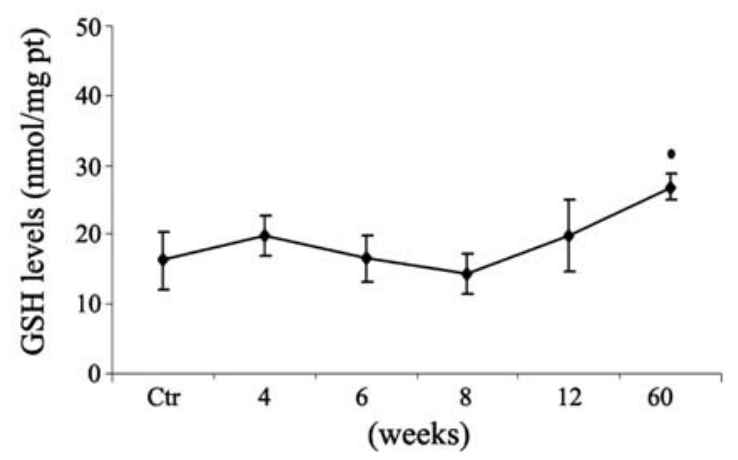

Figure 3. Changes in GSH levels during hepatocarcinogenesis in F344 (A) and $\mathrm{BN}$ (B) rats. The concentrations of GSH were determined by fluorimetric analysis and reported as $\mathrm{nmol} / \mathrm{mg}$ protein. The data are the mean \pm SD from at least three independent experiments. $* \mathrm{p}<0.01 \mathrm{vs}$. Ctr F344; " p $<0.05$ vs. Ctr BN.
Fig. 1B shows a different behaviour of microvessel density in preneoplastic and neoplastic lesions in F344 and BN rats. Notably, microvessel density, evaluated as immunofluorescent staining with anti-VEGF, was clearly lower in BN than F344 rat lesions already at 8 weeks.

On the basis of these observations, we determined the effect of the tumorigenic input with regard to apoptosis and DNA oxidative damage. F344 and BN liver sections (stained as described in Materials and methods) indicated a massive presence of apoptotic nuclei already at 4 weeks, with a progressive increase up to 54 and 60 weeks (Fig. 2A).

8-OHdG immunoreactivity in F344 liver samples was already evident at 4 weeks, thereafter increasing in a timedependent manner. In BN livers, the fluorescence of 8-OHdG was observed only at 60 weeks (Fig. 2B).

The content of hepatic GSH increased in treated F344 rats by 34 and $40 \%$, respectively at 4 and 6 weeks, reaching a 2-fold induction at 54 weeks (Fig. 3A). In BN rats, an increase of GSH by $73 \%$ was observed at 60 weeks (Fig. 3B). No changes in GSSG levels were observed in either rat strain at any time point (data not shown).

Expression levels of SOD1 and SOD2 were comparable in F344 and BN liver lesions and were down-regulated at 54 and 60 weeks, respectively. In particular, SOD1 content decreased by 24 and $63 \%$ in neoplastic lesions of F344 and $\mathrm{BN}$ rats (Fig. 4A). Similarly, SOD2 decreased by 27 and $45 \%$, respectively, in F344 and BN liver lesions (Fig. 4B).
A)

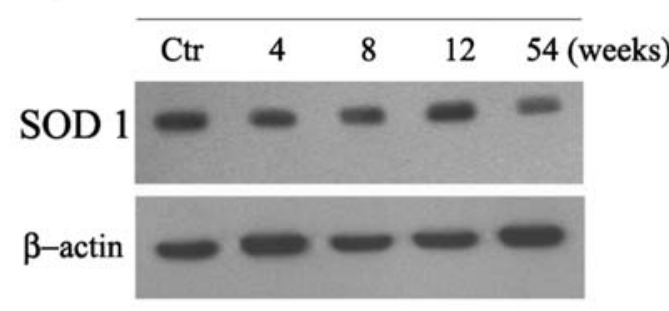

B)

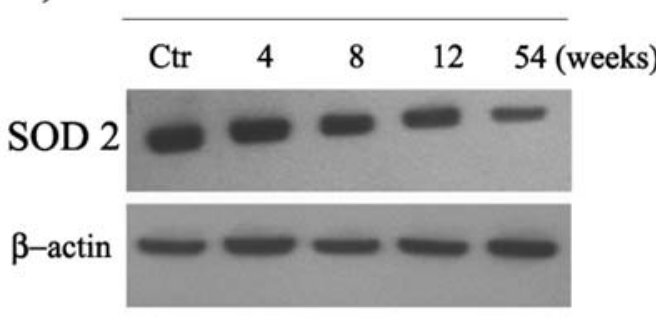

BN

\begin{tabular}{lllll}
\hline Ctr & 4 & 8 & 12 & 60 (weeks)
\end{tabular}
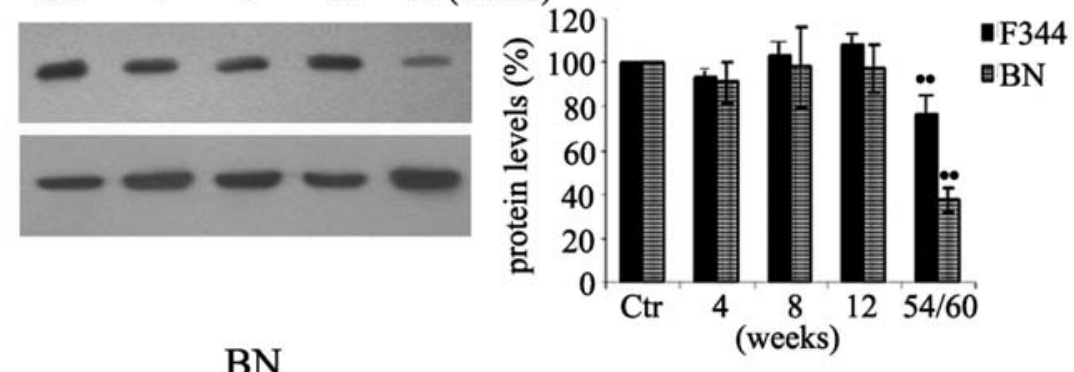

BN

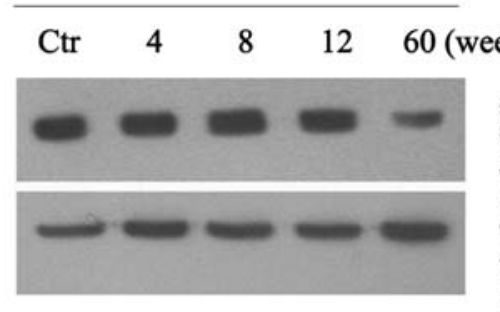

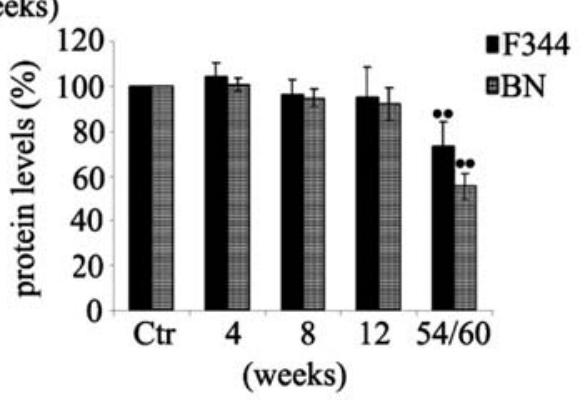

Figure 4. SOD1 (A) and SOD2 (B) expression levels during hepatocarcinogenesis in F344 and BN rats. On the right, histograms summarise quantitative data of means \pm SD of three independent experiments. $\beta$-actin signals represent the immunoblot loading control. “ $p<0.01$ vs. Ctr. 


\section{Discussion}

Fisher 344 (F344) and Brown Norway (BN) rat strains have been demonstrated to be, respectively, susceptible and resistant to hepatocarcinogenesis induced by treatment with DENA/ AAF/partial hepatectomy (9). The resistant phenotype of BN rat strain essentially depends on the incapacity of preneoplastic cells to grow autonomously and evolve to malignancy, associated with a high re-differentiation rate (12). The mechanisms responsible for the acquisition of the resistant phenotype are not completely clear.

In our study, HCC progression is accompanied by the increased expression of VEGF, a crucial regulator of angiogenesis (13). Immunofluorescence analysis shows that the process of new blood vessel formation is less marked in hepatic tissue of $\mathrm{BN}$ rats.

It has been demonstrated that the status of hypoxia, which characterises tumour formation, can stimulate the induction of VEGF which, in turn, stimulates NADPH oxidase to produce reactive oxygen species (ROS) (14). In chronic hepatitis $\mathrm{C}$, infiltration of activated phagocytic cells provides an additional source of ROS production that promotes oxidative stress and damage to proteins, lipids and DNA (15). Recently, it has been shown that oxidative DNA damage is associated with the increased risk of HCC and that hepatic 8-OHdG levels are considered useful markers to identify high-risk patients $(16,17)$. Depending on the extent of DNA damage, cells may undergo apoptosis or proliferate towards tumour progression. The present study indicates that in F344 rat liver lesions, the $8-\mathrm{OHdG}$ positivity, symptom of DNA oxidative damage, is paralleled by the presence of apoptotic nuclei.

On the other hand, in resistant $\mathrm{BN}$ rats, apoptosis is observed during all stages of induction, while DNA oxidation is observed only at 60 weeks, accompanied by attempts of tissue remodelling.

In our context, however, the presence of $8-\mathrm{OHdG}$ and apoptosis in hepatic lesions does not correlate with a depletion of antioxidant GSH, commonly observed in apoptosis (18). The observed enhancement of the GSH content could be interpreted as the liver attempting to counteract the oxidative stress induced by the treatment. Alternatively, it could be attributed to the pleiotropic effects of GSH which controls the onset of tumour cell proliferation $(19,20)$ and is implicated in the detoxification of a wide variety of compounds (21).

Indeed, over-production of GSH has been reported in human cancer (22) and, in line with our findings, Navarro and collaborators also observed that GSH and ROS levels are higher in cancer cells that proliferate actively while GSSG levels remain constant during tumour promotion (23). SOD-1 and SOD-2, respectively cytosolic and mitochondrial antioxidant enzymes, have a variable profile in cancer cells and in particular SOD-2 has been observed to diminish in most tumours compared to the corresponding normal tissues (24-26). Similarly, in our study, the expression levels of SOD1 and SOD2 are importantly down-regulated in neoplastic liver lesions of F344 and BN rats.

Taken as a whole, this study suggests that DNA oxidation might be a necessary step for the progression from dysplastic nodules to HCC. On the other hand, the presence of apoptosis, the enhancement of GSH levels and the down-regulation of SOD expression in both rat strains suggest that neither apoptosis or alterations of the antioxidants, are involved in the acquisition of resistant phenotype. These findings are in line with relevant studies that exclude apoptosis as a mechanism of resistance, attributing a prominent role to re-differentiation (9). In our study, this hypothesis might be supported by a lower DNA oxidative damage detected in neoplastic liver lesions of BN rats. In fact, sound and appreciated literature has hinted that low ROS levels can function as signalling molecules in cell differentiation (27).

However, to better define the correlation between DNA oxidative damage and tumour progression, further studies in hepatocellular carcinoma cell models are required.

\section{Acknowledgements}

We would like to thank Mr Giuseppe Catalano (DIMESUniversity of Genoa) for his technical assistance. This study was funded by grants from G. Gaslini Institute, Genoa University and from the Italian Ministry of Universities: PRIN n² 20077S9A32_002.

\section{References}

1. Farber E and Sarma DS: Hepatocarcinogenesis: a dynamic cellular perspective. Lab Invest 56: 4-22, 1987.

2. De Miglio MR, Virdis P, Calvisi DF, Frau M, Muroni MR, Simile MM, Daino L, Careddu GM, Sanna-Passino E, Pascale RM and Feo F: Mapping a sex hormone-sensitive gene determining female resistance to liver carcinogenesis in a congenic F344.BN-Hcs4 rat. Cancer Res 66: 10384-10390, 2006.

3. Beckman KB and Ames BN: Oxidative decay of DNA. J Biol Chem 272: 19633-19636, 1997.

4. Yamaguchi R, Hirano T, Asami S, Chung MH, Sugita A and Kasai H: Increased 8-hydroxyguanine levels in DNA and its repair activity in rat kidney after administration of a renal carcinogen, ferric nitrilotriacetate. Carcinogenesis 17: 2419-2422, 1996.

5. Nakae D, Kobayashi Y, Akai H, Andoh N, Satoh H, Ohashi K, Tsutsumi $\mathrm{M}$ and Konishi $\mathrm{Y}$ : Involvement of 8-hydroxyguanine formation in the initiation of rat liver carcinogenesis by low dose levels of N-nitrosodiethylamine. Cancer Res 57: 1281-1287, 1997.

6. Hayes JD and McLellan LI: Glutathione and glutathione-dependent enzymes represent a co-ordinately regulated defence against oxidative stress. Free Radic Res 31: 273-300, 1999.

7. Obrador E, Navarro J, Mompo J, Asensi M, Pellicer JA and Estrela JM: Glutathione and the rate of cellular proliferation determine tumour cell sensitivity to tumour necrosis factor in vivo. Biochem J 325 (Pt 1): 183-189, 1997.

8. Johnson F and Giulivi C: Superoxide dismutases and their impact upon human health. Mol Aspects Med 26: 340-352, 2005.

9. Pascale RM, Simile MM, DeMiglio MR, Muroni MR, Gaspa L, Dragani TA and Feo F: The BN rat strain carries dominant hepatocarcinogen resistance loci. Carcinogenesis 17: 1765-1768, 1996.

10. Solt DB, Medline A and Farber E: Rapid emergence of carcinogen-induced hyperplastic lesions in a new model for the sequential analysis of liver carcinogenesis. Am J Pathol 88: 595-618, 1977.

11. Senft AP, Dalton TP and Shertzer HG: Determining glutathione and glutathione disulfide using the fluorescence probe ophthalaldehyde. Anal Biochem 280: 80-86, 2000.

12. Feo F, De Miglio MR, Simile MM, Muroni MR, Calvisi DF, Frau M and Pascale RM: Hepatocellular carcinoma as a complex polygenic disease. Interpretive analysis of recent developments on genetic predisposition. Biochim Biophys Acta 1765: 126-147, 2006.

13. Carmeliet P and Jain RK: Angiogenesis in cancer and other diseases. Nature 407: 249-257, 2000. 
14. Ushio-Fukai M and Nakamura Y: Reactive oxygen species and angiogenesis: NADPH oxidase as target for cancer therapy. Cancer Lett 266: 37-52, 2008.

15. Kaplowitz N: Mechanisms of liver cell injury. J Hepatol 32: 39-47, 2000

16. Tanaka H, Fujita N, Sugimoto R, Urawa N, Horiike S, Kobayashi Y, Iwasa M, Ma N, Kawanishi S, Watanabe S, Kaito $\mathrm{M}$ and Takei Y: Hepatic oxidative DNA damage is associated with increased risk for hepatocellular carcinoma in chronic hepatitis C. Br J Cancer 98: 580-586, 2008.

17. Chuma M, Hige S, Nakanishi M, Ogawa K, Natsuizaka M Yamamoto Y and Asaka M: 8-Hydroxy-2'-deoxy-guanosine is a risk factor for development of hepatocellular carcinoma in patients with chronic hepatitis $\mathrm{C}$ virus infection. J Gastroenterol Hepatol 23: 1431-1436, 2008

18. Ghibelli L, Fanelli C, Rotilio G, Lafavia E, Coppola S, Colussi C, Civitareale $\mathrm{P}$ and Ciriolo MR: Rescue of cells from apoptosis by inhibition of active GSH extrusion. FASEB J 12: 479-486, 1998.

19. Terradez P, Asensi M, Lasso de la Vega MC, Puertes IR, Vina J and Estrela JM: Depletion of tumour glutathione in vivo by buthionine sulphoximine: modulation by the rate of cellular proliferation and inhibition of cancer growth. Biochem J 292 (Pt 2): 477-483, 1993.

20. Friesen C, Kiess Y and Debatin KM: A critical role of glutathione in determining apoptosis sensitivity and resistance in leukemia cells. Cell Death Differ 11 (Suppl. 1): S73-S85, 2004.
21. Shen H, Kauvar L and Tew KD: Importance of glutathione and associated enzymes in drug response. Oncol Res 9: 295-302, 1997.

22. Perquin M, Oster T, Maul A, Froment N, Untereiner M and Bagrel D: The glutathione-related detoxification pathway in the human breast: a highly coordinated system disrupted in the tumour tissues. Cancer Lett 158: 7-16, 2000.

23. Navarro J, Obrador E, Carretero J, Petschen I, Avino J, Perez P and Estrela JM: Changes in glutathione status and the antioxidant system in blood and in cancer cells associate with tumour growth in vivo. Free Radic Biol Med 26: 410-418, 1999.

24. Zhong W, Oberley LW, Oberley TD and St Clair DK: Suppression of the malignant phenotype of human glioma cells by overexpression of manganese superoxide dismutase. Oncogene 14: 481-490, 1997.

25. Oberley LW: Anticancer therapy by overexpression of superoxide dismutase. Antioxid Redox Signal 3: 461-472, 2001.

26. Weydert CJ, Waugh TA, Ritchie JM, Iyer KS, Smith JL, Li L, Spitz DR and Oberley LW: Overexpression of manganese or copper-zinc superoxide dismutase inhibits breast cancer growth. Free Radic Biol Med 41: 226-237, 2006.

27. Owusu-Ansah E and Banerjee U: Reactive oxygen species prime Drosophila haematopoietic progenitors for differentiation. Nature 461: 537-541, 2009. 